\title{
Slow relaxation due to optimization and restructuring: Solution on a hierarchical lattice
}

\author{
János Török ${ }^{(1)}$, Supriya Krishnamurthy ${ }^{(2, *)}$, János Kertész ${ }^{(1)}$ and Stéphane Roux ${ }^{(3)}$ \\ (1): Department of Theoretical Physics, Institute of Physics, \\ Budapest University of Technology and Economics, 8 Budafoki út, H-1111 Budapest, Hungary \\ (2): Department of Theoretical Physics, University of Oxford, 1 Keble Road, OX1 3NP, UK \\ (3): Surface du Verre et Interfaces, UMR CNRS/Saint-Gobain, \\ 39 Quai Lucien Lefranc, 93303 Aubervilliers Cedex, France ${ }^{*}$
}

(Dated: November 20, 2018)

\begin{abstract}
Motivated by the large strain shear of loose granular materials we introduced a model which consists of consecutive optimization and restructuring steps leading to a self organization of a density field. The extensive connections to other models of statistical phyics are discussed. We investigate our model on a hierarchical lattice which allows an exact asymptotic renormalization treatment. A surprisingly close analogy is observed between the simulation results on the regular and the hierarchical lattices. The dynamics is characterized by the breakdown of ergodicity, by unusual system size effects in the development of the average density as well as by the age distribution, the latter showing multifractal properties.
\end{abstract}

\section{INTRODUCTION}

Slow dynamics with no separation of time scales represent a major challenge of statistical physics. Experimental or simulation approaches are extremely difficult, so in most cases new ideas and models are needed for the understanding of this kind of problems.

There can be different roots of slow dynamics: Systems close to the critical point slow down enormously due to the increasing characteristic time. Phase separation is often accompanied by a slow coarsening process [1]. In glasses the free energy landscape is so complicated and structured that the system never finds the global minimum and shows a history dependent behaviour called aging [2]. Slow dynamics may also occur in intrinsically dynamic, driven systems leading to scale free fractal structures. The name of self-organized criticality covers a whole family of related models [3].

In this paper we study a model (introduced in [1]), where the system exhibits a very slow evolution with a tendency of getting stuck in metastable states. However the model is different from those studied earlier in the sense that there is an element of both energy as well as entropy barriers being present as a result of the rules of evolution. We are able to directly link the slow evolution to a break down of ergodicity in the dynamics. This then leads to several interesting features of the model such as non-trivial system size effects, a multi-fractal "age" distribution and a non-trivial temporal evolution.

Motivated by our study of shearing loose granular materials [1], we report in this paper about a new mechanism leading to slow dynamics. In granular materials displacement occurs in a localized manner, in 'shear bands' which are formed along the weakest parts of the

*present address: Santa Fe Institute, 1399 Hyde Park Road, Santa Fe NM 87501 samples. During shear, grains can rearrange themselves and occasionally strengthen the local structure. In such a case, the shear band finds a new configuration which avoids this zone. Based on this picture we introduced a model where consecutive steps of optimization (finding the weakest part of the sample) and restructuring (random rearrangement of grains) takes place. Assuming translational invariance in the shear direction the model becomes two-dimensional. We have studied the model numerically in detail on regular lattices [5], however, it is difficult to go beyond the simple description of numerical simulations. It turned out to show unexpected properties including extremely slow dynamics and unusual size dependence (breakdown of ergodicity), and it provides with interesting predictions for the granular system.

The aim of this paper is to study this same model on a hierarchical diamond lattice both numerically and analytically, and to compare these results with the simulations on the euclidean lattice. We find that despite the very different connectivities of these two lattices, the qualitative behaviour is much the same; for some properties, there is a quantitative matching as well. The recursive nature of the hierarchical lattice however aids the analytical treatment greatly, thus helping us getting a deeper understanding of the problem.

The paper is organized as follows: In the next section we define the model in general and on the hierarchical lattice. In Section III, the relation of the model to other problems of statistical physics is discussed. In Section IV, the numerical results are shown and compared to the regular lattice simulations. In Section $\mathrm{V}$ we present the exact asymptotic solution of the model. We conclude in section VI. Appendix A and B contain technical details of the calculations used in Section $\mathrm{V}$. 


\section{THE MODEL AND THE HIERARCHICAL LATTICE}

The model that we study in this paper is defined as follows: A two-dimensional field is characterized by a single scalar parameter, the density $\varrho(x, y)$. Initially this density is generated randomly from the distribution $p_{i}(\varrho)$. At every step we search for the minimal path $\mathcal{P}^{*}$ that is defined as follows: The minimal path is a continuous, directed path $\mathcal{P}$ that spans the system in the $x$ direction and the sum $S$ of the local densities along it,

$$
S(\mathcal{P})=\sum_{(x, y) \in \mathcal{P}} \varrho(x, y)
$$

is minimal among all possible paths. The minimal path is the path $\mathcal{P}^{*}$ for which $S\left(\mathcal{P}^{*}\right)$ is minimum.

Once the minimal path is found the density values of the points belonging to the minimal path $(x, y) \in \mathcal{P}^{*}$ are replaced by new densities randomly picked from the distribution $p_{r}(\varrho)$.

The above process is repeated as long as desired. A single time step consists of both searching for the minimal path, as well as refreshing the local densities along it.

In the following we restrict ourself to the case where $p_{i}$ and $p_{r}$ are uniform distributions in the interval $[0: 1]$. Our model is discretized on a lattice. In [5] we report on detailed numerical results for various properties of the model on the Euclidean square lattice. The analytic treatment on the square lattice has not been possible so far. However, in this paper, we obtain exact asymptotic solutions for the model on the hierarchical diamond lattice.

The hierarchical diamond lattice [7] is constructed as follows: We first consider a single bond connecting two points $\mathrm{A}$ and $\mathrm{B}$. This constitutes the most elementary (generation 0) lattice. The first generation lattice is obtained by substituting the unique bond by an elementary "diamond" of four bonds, i.e. two parallel connections each consisting of two bonds in series [Fig. 1 b)]. The next generation is obtained recursively by the substitution of each bond by a diamond [Fig. 11 c)]. Repeating the above procedure $N$ times, produces a $N$ th-generation hierarchical lattice. This lattice has a dimension equal to 2 , and hence can be compared to its Euclidian counterpart.

All results are based on the exploitation of the recursivity of the construction of the lattice. If one can compute the properties of an elementary diamond and transform this into a single bond endowed with the same, a recursive use of this procedure clearly allows the reduction of the entire lattice back to a single bond thus determining the global behavior. This is a real space renormalization procedure and the structure of the lattice makes such renormalization treatments exact. Hierarchical lattices have been widely used to study several phenomena such as percolation [8], spin models [9], sums of directed paths [10] etc. However, usually there is a price to pay in that the result may differ from its Euclidean lattice counterpart. There is no general formalism by which means to

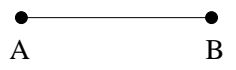

a)

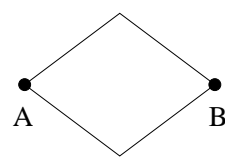

b)

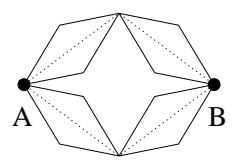

c)
FIG. 1: The 0th a), 1st b) and 2nd c) generations of the hierarchical lattice.

estimate the validity of hierarchical lattice results for the Euclidean lattice. Therefore, it is necessary to resort to numerical results to assess the similarity between the two cases. It will be shown in the following that indeed the analogy between the results obtained on both kinds of lattices is extremely close. Therefore, the analytical solution obtained here provides a better understanding of the Euclidean lattice case.

\section{RELATION TO OTHER STATISTICAL PHYSICS MODELS}

Before reporting the result of numerical simulations on the hierarchical lattice, we point out some analogies which can be drawn between our model and other diverse problems of statistical physics.

The rules of our model, finding the extremal directed spanning path at every instant, is similar to finding the ground state of a directed polymer in a random potential [11. However, in our case this potential is uncorrelated only at the beginning; it changes in time through the process already described above, of ascribing new densities to all sites along the minimal path. The shape of the path on Euclidean lattices is found to be self-affine in the directed polymer problems. The model studied here changes the underlying potential landscape in a selforganized way and naturally induces correlations. These in turn change the self-affine exponent of the path. This feature is studied numerically in an earlier paper [5].

The rules of our model can be regarded as a generalization of the Bak-Sneppen model of evolution [12], but in higher dimensions. Indeed, the constraint of finding the minimal path and then changing it, puts this model in a class of extremal models studied in contexts as different as interface depinning [13] and flux creep [14]. However there is an important difference between our model and other extremal models. In the latter case, the system (usually one-dimensional) reaches a steady state which is 'self-organized critical', in the sense that there is a powerlaw distribution for avalanches in the steady state. In the case of our model, no steady state is reached and all quantities depend on time. As we will see, we can define avalanches which are indeed power-law distributed, but always with time dependent prefactors. The difference is best illustrated if we look at the simplest of these extremal models, the Bak-Sneppen model [12. This model is defined on a one dimensional periodic array of ran- 
dom numbers where at every update, the least and its neighbours are refreshed from a given time independent probability distribution. Our model is however related to a variant of this in which only the least is changed. In one dimension, changing only the least does not lead to a very interesting behaviour. However in two dimensions, as we will see, changing only the minimal path leads to very non-trivial effects. Further, the simple minded variation of the original Bak-Sneppen model turns out to be very useful in solving our model on the hierarchical lattice.

There are also connections between this model and the apparently unrelated problem of a random walk in a disordered potential. If we consider a one-dimensional crosssection of the model perpendicular to the minimal path, we could imagine the point through which the interface passes through, as the position of a random walker. The subsequent dynamics can then be interpreted as that of a walker moving through an initially random potential, modifying it along the way. While the actual dynamics of the interface in two-dimensions is quite complicated to translate in its entirety into one-dimension, it is possible to do so in the simplest case when only corner flips are allowed for the interface. For the walker, this simply translates to the condition that the subsequent position of the walker is on one of the neighbouring sites of the present one, chosen by an inequality condition. The value at the site the walker has just left, is also changed. We have studied such an active walker model in detail 15 and find that it leads to logarithmically slow dynamics.

Because of the extremal condition used in finding the minimal path at each time step, the solution of the model on the hierarchical lattice uses results from extreme-value statistics [16]. We also find that the "age" distribution, i.e. the probability distribution of the number of times up to time $T$, that a given site has been a part of the shear band (and has hence been changed), has many similarities with models of fragmentation studied in various contexts [17].

There has been recently an upsurge of interest in systems exhibiting an anomalously slow relaxation. Such a behavior is generically reminiscent of a glassy behavior, and this analogy has motivated a number of studies [18]. Just to mention one example related to granular media, the slow compaction of sand under repeated tapping 19, 20 displays analogies with glasses obtained at different cooling rates. A number of different modelings of this compaction process have been proposed [6, 18, 21, 22, 23, 24, 25, 26, 27, 28, 29, 30, 31, 32. Some of these models emphasize the role of a broad distribution of energy barriers which have to be overcome through thermal activation. This naturally leads to the occurrence of a wide distribution of characteristic times, with a slower and slower dynamics as the easiest barriers are exhausted. Models of this sort have been looked at in a wide variety of contexts ranging from trap models [33] and anomalous diffusion in the presence of quenched disorder [34] to constrained spin systems 335], granular compaction [6] and ageing in soft solids [36]. Other ap- proaches put more emphasis on the collective nature of the necessary rearrangement allowing for a relaxation $118,21,22,23,24,25,26,27,28,29,30,31,32$. As time passes, the relaxation has to become more and more cooperative, and hence the barrier is more entropic than energetic. Models with entropic barriers have been well studied in other contexts too, such as the Backgammon Model [37].

In the model we study in this paper, as we shall see, we observe a very slow dynamics which can indeed be compared to such glassy behavior. We do not include any temperature stricto sensu, however, the randomness of the local densities can in some way be compared to thermal noise. The crude classification we proposed above between energetic and entropic emphasis is not quite suited to our model, where both aspects are simultaneously present. The necessary cooperative nature of efficient events is included in the search for a minimal path where all sites contribute with the same weight. However, a local dense configuration can occur at any time, and remain quenched thereafter for very long. This is like an energy barrier since in order for the minimal path to go through this region, all minimal paths with smaller energies need to be eliminated This is thus a very rare event with the probability becoming smaller and smaller as time passes.

To push forward the analogy with a glassy system, we will see that we observe a breakdown of ergodicity, in the sense that the activity is not spread uniformly throughout the system. Hence if we partition a system into two sub parts (even for large sizes), the relative "age" of the two subsystems will tend to a broad distribution, and not to a narrow one as expected for homogeneous systems. This implies that the scaling of the compaction in both space and time is expected to be non trivial.

\section{NUMERICAL RESULTS}

In this section we present briefly the most important numerical results on both the hierarchical and Euclidean lattices.

The most important quantity of the system is the average density. We define it as the mean density of all sites not belonging to the minimal path and we denote it by $\langle\varrho\rangle(t)$. The importance of not including the minimal path in the average density is that this definition ensures that $\langle\varrho\rangle$ monotonically increases with time. Furthermore, as we will see, at late times the minimal path mostly remains unchanged. Since we keep refreshing the same bonds again and again, the density along the minimal path is simply taken from the known distribution $p_{r}(\varrho)$ and there is no need to incorporate this into $\langle\varrho\rangle$.

In our case, as $p_{i}=p_{r}$ is a uniform distribution between 0 and 1 , it is clear from the rules that the system evolves towards the limiting state of $\varrho(x, y)=1$ everywhere. It is natural thus to plot $1-\langle\varrho\rangle(t)$ as done on Fig. 2. 

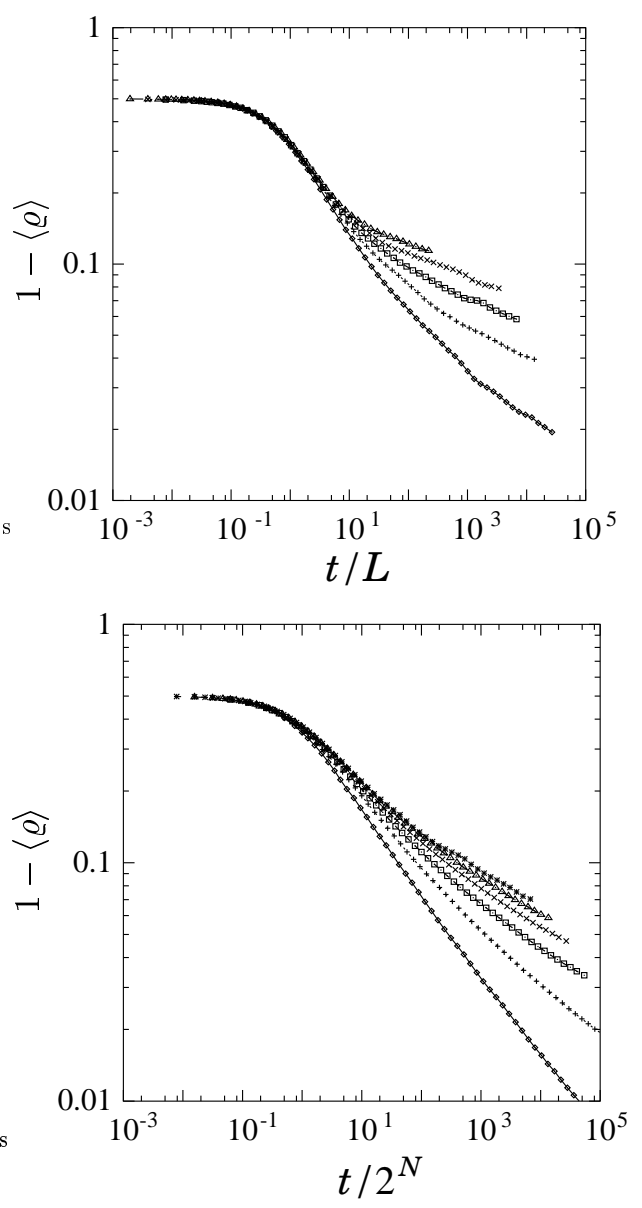

FIG. 2: The difference of the average density from its asymptotic value 1 as a function of time. The system sizes are $L=32,64,128,256,512$ for the square lattice (upper) and $N=2$ to $N=7$ for the hierarchical lattice (lower) from bottom to top respectively. The average was done over all the inactive sites in the lattice and for an ensemble of 20 to 1000 samples. System size increases from bottom to top in both cases.

On Fig. 2 we present both the $L \times L$ square (upper) and hierarchical (lower) numerical results for $\langle\varrho\rangle(t)$. We can make two immediate observations: The $t / L\left(t / 2^{N}\right)$ scaling works nicely up to about unity after which a system size dependent relaxation is observed which is slower for larger systems. The density decay seems to be slower than any power-law. The other quantity that we study in detail is the Hamming distance, i.e., the number of different bonds between consecutive minimal paths. We denote this quantity by $d$. The value of $d$ may vary from 0 to $L\left(2^{N}\right)$.

As can be seen in Fig. 3, in both lattices, at an early stage the mean Hamming distance is close to the system size (i.e. two consecutive paths do not overlap at all). It then decreases monotonically to 0 . We recall that when the distance is equal to 0 , then the two successive conformations of the minimal path are identical, in spite of the total renewal of random densities along them. This
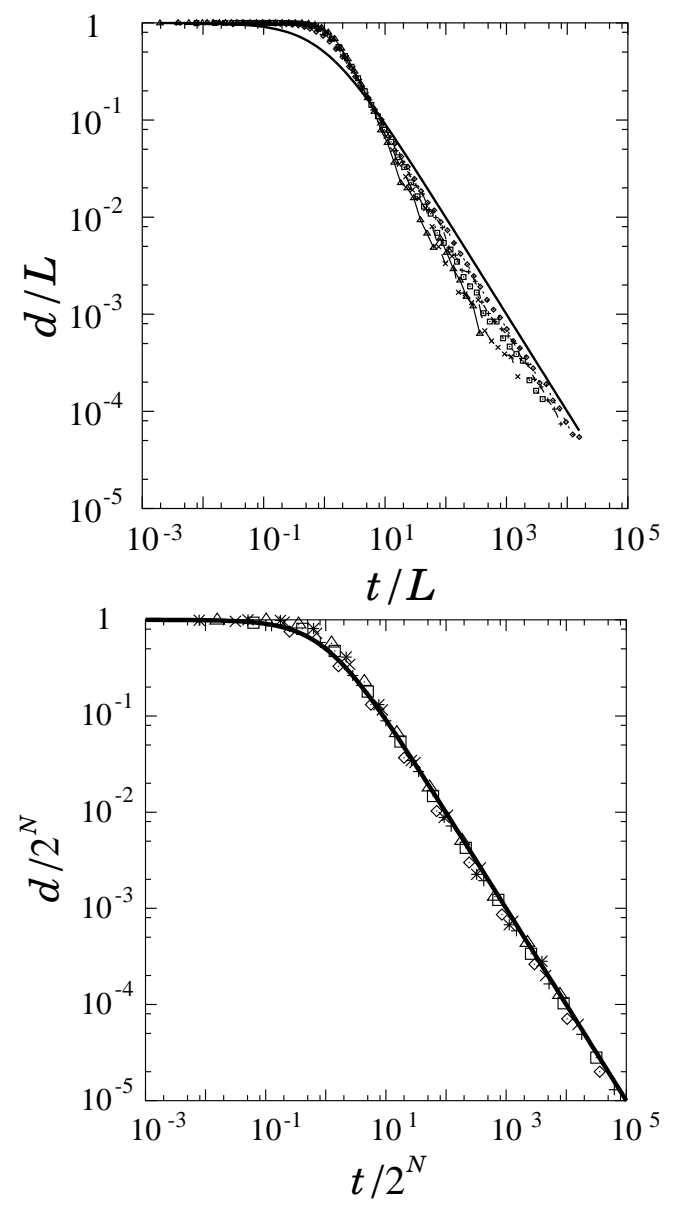

FIG. 3: The average Hamming distance versus time for square (upper) and hierarchical lattice (lower). The same system sizes were scaled together as on Fig. 2. On both figures, the analytical prediction $1 /(t+1)$ is plotted over the data. Note that scaling with system size is excellent for the hierarchical lattice while it seems to display systematic corrections for the Euclidean case.

indicates that minimal paths have a tendency to remain more and more persistent as the system "ages".

\section{ANALYTICAL RESULTS ON THE HIERARCHICAL DIAMOND LATTICE}

In the following we show how some of the above listed properties of our model can be understood analytically on the hierarchical lattice. The techniques we use are essentially those of extreme value statistics.

\section{A. Summary of the solution}

We use the hierarchical nature of the diamond lattice to calculate the average density for level $N$ knowing the results on level $N-1$. 
Let us introduce the following notations: The average density of the inactive sites in a (sub)lattice level $N$ is $\varrho^{(N)}(t)$, the density of the active sites is $x^{(N)}(t)$. In (sub)lattices we define the active site to be the minimal path of this (sub)lattice regardless of whether it is a part of the global minimal path or not. We will use the indices $l, r, u, d$ for left, right, up, down respectively to indicate the parts of a diamond [(sub)lattice] corresponding to the illustration on Fig. 1 1 b,c).

A generation $N$ lattice is constructed by putting together 4 generation $N-1$ sublattices (see Fig. 1) with two series couplings and one parallel coupling. The series coupling of the sublattices is easily taken into account, both the density of the minimal path as well as the density of the sites in the bulk are simply averaged:

$$
\begin{aligned}
& x^{(S(N-1))}(t)=\frac{1}{2}\left(x_{l}^{(N-1)}(t)+x_{r}^{(N-1)}(t)\right)=x^{(N-1)}(t) \\
& \varrho^{(S(N-1))}(t)=\frac{1}{2}\left(\varrho_{l}^{(N-1)}(t)+\varrho_{r}^{(N-1)}(t)\right)=\varrho^{(N-1)}(t)
\end{aligned}
$$

where the superscript $S$ refers to two systems coupled in series.

The next step is the coupling in parallel of two series couplets. The density of the shear band is simply the minimum of that of the two subsystems. The average density of the bulk contains two contributions: the average densities of the subsystems as well as the average density of one of the active paths (the one which is the larger of the two contenders for the global minimal path).

$$
\begin{aligned}
x^{(N)}(t) & =\min \left\{x_{u}^{S(N-1)}\left(t_{u}\right), x_{d}^{S(N-1)}\left(t_{d}\right)\right\} \\
\varrho^{(N)}(t) & =\frac{\left(4^{N}-2^{N+1}\right)}{2\left(4^{N}-2^{N}\right)}\left\{\varrho_{u}^{S(N-1)}\left(t_{u}\right)+\varrho_{d}^{S(N-1)}\left(t_{d}\right)\right\} \\
& +\frac{2^{N}}{\left(4^{N}-2^{N}\right)} \max \left\{x_{u}^{S(N-1)}\left(t_{u}\right), x_{d}^{S(N-1)}\left(t_{d}\right)\right\}
\end{aligned}
$$

where $4^{N}$ is the total number of bonds, $2^{N}$ is the number of the bonds in a path on a generation $N$ lattice and the multiplicative factors in the above equation are the appropriate fractions of bonds at generations $N$ (see Fig. 1).

There is a further subtlety here. The time $t$ counts the total number of updates at generation $N$. However the "time" relevant for a subsystem at generation $N-1$ is simply the number of times the subsystem itself has been updated. Since only one of the two systems in parallel is updated at every instant, the "age" of a subsystem at level $N-1$ is less than $t$ and is denoted by $t_{u}$ and $t_{d}$ in the above equation. In Appendix A we prove that the relative age of either subsystems $t_{u} / t$ or $t_{d} / t$ is uniformly distributed between 0 and 1 in the limit of a large time $t$. Thus we rewrite the second equation of (3)

$$
\begin{aligned}
& \varrho^{(N)}(t)=\frac{\left(4^{N}-2^{N+1}\right)}{\left(4^{N}-2^{N}\right)} \frac{1}{t} \int_{0}^{t} \varrho^{(N-1)}\left(t^{\prime}\right) \mathrm{d} t^{\prime}+ \\
& +\frac{2^{N}}{\left(4^{N}-2^{N}\right)} \frac{1}{t} \int_{0}^{t} \max \left(x^{(N-1)}\left(t^{\prime}\right), x^{(N-1)}\left(t-t^{\prime}\right)\right) \mathrm{d} t^{\prime} .
\end{aligned}
$$

The second term in Eq. 10 comes from the competition between the minimal paths in the two subsystems coupled in parallel. Only one of these is the global minimum and the larger has hence to be incorporated into the density of the system. At every timestep that a subsystem is updated the minimal path of that subsystem can switch to either side. Since the two parallel subsystems are entirely disjunct the path changes sides if the mean of the random numbers generated along the minimal path is larger than the minimal path in the other subsystem. This competition is present at all levels of the hierarchy.

The problem of the minimal path in the parallel coupling can be thus described by a simple model that we call the two site model. The two site model is defined as follows: There are two sites, each with a single value generated by a random number drawn from a given distribution $p(x)$ (in our case at level $N$ it is the sum of $2^{N}$ independent random numbers each of which is taken from the uniform distribution between 0 and 1 ). We choose the site with the smaller value and refresh it with a random number generated from the same distribution [38]. The dynamics consists of repeating this procedure. Important features of this problem turn out not to depend on the distribution $p(x)$, since the entire evolution is only based on the ordering of the values. As a result one can map any bounded distribution onto a uniform one and preserve the same history of the activity. It is thus easy to deduce that the probability of having an active site in one subsystem for a given time, knowing the age of the system, is independent of $p$. We present in Appendix $\mathrm{B}$ an analytical derivation of relevant properties of this problem.

\section{B. Age distribution}

We have seen that as a result of the parallel coupling, the time spent in one subsystem, or the "age" of a subsystem, $\theta$, differs from the actual time $t$ and that $\theta / t$ is uniformly distributed between 0 and 1 in the two-site model. Repeating the above argument from the entire system down to a single bond, we can extract the statistical distribution of ages relative to the total time.

Let $p_{N}(T ; t)$ be the statistical distribution that a given bond was updated exactly $T$ times at time $t$ in a lattice of generation $N$. Using the above argument, we can relate these distributions of different generations through the relation:

$$
p_{N+1}(T ; t)=\int_{T}^{t} \frac{p_{N}\left(t^{\prime} ; t\right)}{t^{\prime}} \mathrm{d} t^{\prime}
$$


with $p_{0}(T ; t)=\delta(t-T)$ and $N \geq 1$. In other words the age of the bonds or subsystems can be obtained by a "fragmentation" process: At every level to get the age of the upper and lower arms in the parallel coupling the age of the diamond is cut into two pieces with a uniform distribution. Not surprisingly equations similar to the above are well known in the context of models of fragmentation [17.

The solution of the above recursion is :

$$
p_{N}(T ; t)=\frac{1}{t} \frac{(\log (t / T))^{N-1}}{(N-1) !}
$$

for $T \leq t$. Introducing the relative age $\theta=T / t$, we observe that the above distribution becomes independent of the time $t$ (the $1 / t$ prefactor is absorbed in the measure $d \theta=d T / t)$.

We note here that models of fragmentation which are described by similar equations usually look for a steady state solution i.e. an $N$ independent solution at late times. However, in our case, as explained below, the $N$ dependence is crucial and has necessarily to be kept. Further the order in which $N$ and $t$ are taken to infinity is very important as well.

It is interesting to note that the above distribution can be simply expressed in the framework of multifractality, which was introduced to characterize the scale dependence of statistical distributions. This analysis naturally provides a generalized "dimensional analysis" of a local quantity $x$, with a distribution $p_{L}(x)$. We introduce the scaling index $\alpha$ and associated fractal dimension $f(\alpha)$ of the support of the set of $x$ values defined through

$$
\begin{cases}x & \propto L^{\alpha} \\ x p_{L}(x) & \propto L^{f(\alpha)-d}\end{cases}
$$

where $d$ is the space dimension. Alternatively $\alpha=$ $\log (x) / \log (L)$ and $f(\alpha)=d+\log \left(x p_{L}(x)\right) / \log (L)$. In our case, the local quantity $x$ is the relative age $\theta=T / t$ and $d=2$, thus

$$
\begin{aligned}
\alpha= & \frac{\log (\theta)}{\log (L)} \\
f(\alpha)= & 2+\alpha+\left(\frac{1}{\log (2)}-\frac{1}{\log (L)}\right)\{\log (-\alpha)+ \\
& \left.+1+\log (\log (2))-\frac{\log (\log (L / 2))}{\log (L)}\right\}
\end{aligned}
$$

where we have used the Stirling formula, assuming $2^{N}=$ $L \gg 1$. In this limit, we have

$$
\begin{aligned}
f(\alpha)= & \alpha+\frac{\log (-\alpha)}{\log (2)}+\frac{1+2 \log (2)+\log (\log (2))}{\log (2)}+ \\
& +\mathcal{O}\left(\frac{1}{\log (L)}\right)
\end{aligned}
$$

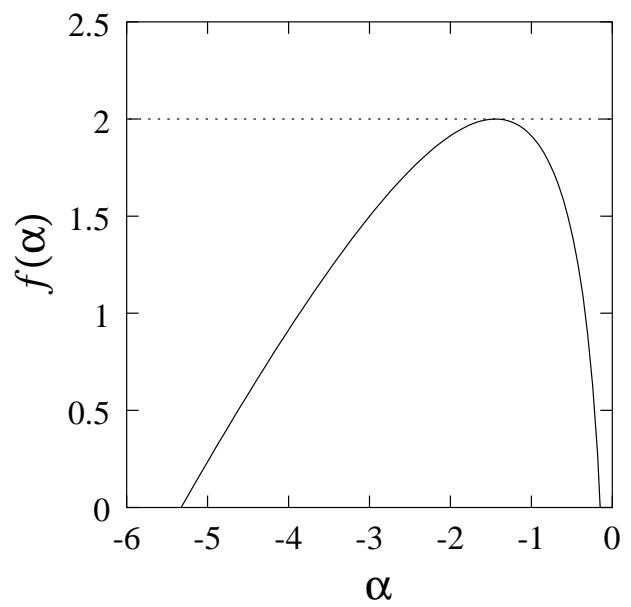

FIG. 4: Multifractal spectrum of the (relative) age distribution in the hierarchical lattice. $\alpha$ gives the scaling exponent of the age with the system size, and $f$ the corresponding fractal dimension of the support of the set of sites contributing to a given $\alpha$.

where in the limit of an infinite system size, $L \rightarrow \infty$, the correction term $\mathcal{O}(1 / \log (L))$ vanishes. Due to this formalism we arrive at a system size independent description of the distribution of relative age, although the distribution itself depends on $L$. Moreover, the interpretation of the formalism is rather natural. The subset of sites whose age scales as a power-law of the system size $\theta \propto L^{\alpha}$ has a fractal dimension $f(\alpha)$.

Figure 1 shows the asymptotic form of the multifractal spectrum. The range of $\alpha$ values corresponding to a positive fractal dimension is $\alpha_{\min } \approx-5.33$ and $\alpha_{\max } \approx$ -0.15 . The scaling exponent characterizing the maximum number of sites $\alpha_{0}$ is the one for which $f$ is maximum, i.e. $f=2$, and hence $\alpha_{0}=-1 / \log (2) \approx-1.44$. Let us emphasize that this description is only valid for very large times. Otherwise, the finite cut-off in the time distribution will affect the multifractal spectrum. Moreover, we have discarded correction terms which will disappear as $1 / \log (L)$, i.e. very slowly. This may render this spectrum difficult to observe numerically.

This analysis shows that the relative age $\theta$ does not scale with $L$ in a unique fashion. When computing a moment of order $m$, only one scaling set dominates. The precise value of this dominant $\alpha$ depends on $m$. It corresponds to the condition $\mathrm{d} f(\alpha) / \mathrm{d} \alpha=-m$ or

$$
\alpha(m)=\frac{-1}{(m+1) \log (2)}
$$

unless the corresponding value of $f$ is negative. The moment then varies as

$$
\left\langle\theta^{m}\right\rangle \propto L^{\tau(m)}
$$

where

$$
\tau(m)=f(\alpha(m))-d+m \alpha(m)
$$


In our example,

$$
\tau(m)=-\frac{\log (m+1)}{\log (2)} .
$$

\section{Average density}

Now we can use the above results to get the final form of the time evolution of the average density.

The recursion relation in Eq. 4 is composed of two terms. Let us study the first term. As the integration operation is additive we can consider separately all the components of a lattice of generation $N$; from subsystems of generation $N-1$ right upto individual bonds.

First we consider the generation 1 lattice, the simple diamond. The two site model gives the exact time dependence of the inactive bonds in the asymptotic limit (see Appendix $\mathrm{B}$ ) which is $1-B^{(2)}(t) \propto 1 / \sqrt{t}$. In order to get the contribution of these bonds we have to complete the integral of Eq. 4 , the expectation value of $T^{-1 / 2}$, with the correct "age" distribution of these subsystems. Note that we calculate the age of a diamond (level 1 object) not a bond. Therefore in a level $N$ system we shall use $p_{N-1}(T ; t)$ for the age distribution.

$$
\begin{aligned}
1-\left\langle\varrho_{1}\right\rangle & \propto t^{-1 / 2} \int_{0}^{1} \theta^{1 / 2} p_{N-1}(T ; t) \mathrm{d} \log (\theta) \\
& =t^{-1 / 2} \int_{0}^{1} \theta^{1 / 2} \frac{(-\log (\theta))^{N-2}}{(N-2) !} \mathrm{d} \log (\theta) \\
& =t^{-1 / 2} 2^{N-1} \int_{0}^{\infty} \frac{x^{N-2}}{(N-2) !} \exp (-x) \mathrm{d} x \\
& =t^{-1 / 2} L / 2
\end{aligned}
$$

where $\left\langle\varrho_{i}\right\rangle$ is the contribution of sublattices of generation $i$ to the average density.

The above result has two important implications. First, the time dependence of the sublattice, $1 / \sqrt{t}$, is preserved on the global scale. Secondly, the statistical distribution of ages gives rise to a system size dependence, i.e. a power-law of $L$, which in the above example displays a trivial exponent 1 . More generally, this exponent is $\tau(-1 / 2)$ as derived above.

The above expression accounts for about half of the bonds. The next term which enters in the coupling is the inactive minimal paths in the level 2 subsystems (Fig. 1 c). The length of these paths is $2^{2}=4$ bonds. The twosite model predicts an asymptotic $t^{-1 / 4}$ time dependence for $1-B^{(4)}(t)$. Thus here we have to use the moment of order $-1 / 4$ and the age at generation $N-2$ :

$$
\begin{aligned}
1-\left\langle\varrho_{2}\right\rangle & \propto t^{-1 / 4} \int_{0}^{1} \theta^{1 / 4} p_{N-2}(T ; t) \mathrm{d} \log (\theta) \\
& =t^{-1 / 4}(4 / 3)^{N-2} \int_{0}^{\infty} \frac{x^{N-3}}{(N-3) !} \exp (-x) \mathrm{d} x \\
& =t^{-1 / 4}(3 / 4)^{2} L^{\log (4 / 3) / \log (2)}
\end{aligned}
$$

We see in this example a non-trivial scaling with the system size and a slower time dependece.

We can carry out this same procedure for higher genration of subsystems. The length of the path in a level $i$ sublattice is $n=2^{i}$ thus their contribution is:

$$
1-\left\langle\varrho_{i}\right\rangle \propto t^{-1 / n}(1-1 / n)^{i} L^{-\log (1-1 / n) / \log (2)}
$$

Thus we observe that the scaling of the mean density can be cast into the form of a sum of power-laws with a vanishing exponent $1 / L=2^{-N}$ and thus a slower and slower decay to zero. Each of these terms has a prefactor which exhibits a different scaling with $L$, and hence, the aging of different system sizes cannot be accounted for by a simple reduced time such as $t / L$. The latter only holds for the first subset (half of the system size), and not the successive hierarchy of minimal paths. This argument explains why the time evolution of the mean density seemed to follow a unique curve when plotted as a function of $t / L$ for early times. However, as time increases, we note a breakdown of this simple scaling, and larger systems shows a slower and slower increase in the average density. It is interesting to note however that for (extremely) large times, (i.e. vanishing exponent $1 / n)$, the moment will depend only on the combination $t / L^{1 / \log (2)}$. This exponent which appears in $L$ is the $\alpha$ value of the largest fractal dimension, $f=2, \alpha=\alpha_{0}$ in the multifractal spectrum.

The above analysis is, however, valid only for very late times, after a long transient. The sum of $m$ identically distributed random variables (when each individual variable is taken from the uniform distribution) can be approximated by a power-law distribution, only at late times. Before that, it is well approximated by a Gaussian, by the central limit theorem. It is only when we are pushed to the tails of the distribution that the power-law regime occurs. However the extremising rule makes this inevitable, though after a long transient. For instance for a generation 3 minimal path, consisting of 8 bonds in series, this transient ageing period lasts for about $t \sim 8 ! \approx 40000$ time steps. $t$ thus has to be much larger than this so that the lower limit of the integral in Eq. 14 can be taken to 0 . We see that our computation becomes strictly applicable only for extremely late times.

Finally we put together all the information we have on the increase of the density of the inactive bonds of the hierarchical lattice i.e.:

1. The lattice is a collection of two site systems from level $1 \leq i \leq N$ where the "ageing" of a bond in any level is given by Eq. B7.

2. The number of bonds in each level gives a prefactor of $2^{N-i} /\left(2^{N}-1\right)$.

3. The age distribution at each level results in an additional factor as in Eq. 16.

We finally get the following result for the average density: 


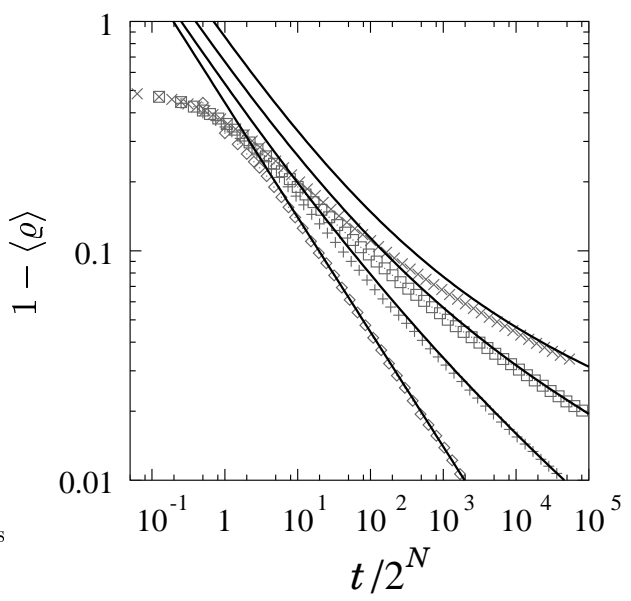

FIG. 5: The test of the analytical result. The numerical data are plotted with symbols the corresponding analytical with solid lines. The hierarchical level is $N=1,2,3,4$ from bottom to top respectively.

$$
\varrho^{(N)}(t)=1-\sum_{i=1}^{N}\left(\frac{2^{N-i}}{2^{N}-1}\right) \frac{a(i) L^{z(i)}}{(t)^{b(i)}}
$$

where (using $n=2^{i}$ and $L=2^{N}$ for a lattice of level $N$ ):

$$
\begin{aligned}
& a(i)=\Gamma(1 / n)(n !)^{1 / n} n^{-2}(1-1 / n)^{i} \\
& b(i)=1 / n \\
& z(i)=-\frac{\log (1-1 / n)}{\log (2)}
\end{aligned}
$$

Figure 目 visualizes this result compared to the numerical data.

\section{Intermediate time behavior of the average density}

For a minimal path of length $\ell=2^{n}$, we are interested in the maximum value of the sum of $\ell$ random numbers over a number of realizations equal to the age $t$. For large $\ell$, the distribution of the average element in the sum converges towards a Gaussian of average $1 / 2$ and standard deviation $1 / \sqrt{12 \ell}$. The expectation value of the largest such element over a time $t$ is thus such that

$$
\int_{S}^{\infty} \frac{2 \sqrt{3 \ell}}{\sqrt{2 \pi}} \exp \left(-6 \ell(x-1 / 2)^{2}\right) \mathrm{d} x \approx \frac{1}{t}
$$

It is important to note that this expression is valid for large $\ell$ and moderate $t$, whereas we previously considered the limit of large $t$ and moderate $L$. The order of the limits plays a crucial role. The mean value of the densities along the minimal path thus departs only very slowly from $1 / 2$. This slow change of the density of the sites along the minimal path in turn plays a crucial role in the very slow decay of the mean density in spite of the vanishing fraction of bonds involved. The departure from $1 / 2$ varies roughly as $\sqrt{\log (t)}$. Taking such a form into account, we see that the average density does not converge to 1 any longer, just as if some bonds were quenched close to their average value $1 / 2$, up to a very slowly evolving correction. Thus numerically, one can achieve a reasonable fit of the evolution of $\varrho$ to values different from 1 . However, as the time window is enlarged, the effective asymptotic $\varrho$ increases. Reciprocally, extending the system size, this asymptote decreases. Thus, in spite of the quality of the fits which can be produced this way, we underline the fact that such an approach is only applicable to a fixed time or system size window.

\section{E. Hamming distance}

Let us now consider the overlap function shown in Fig. 3. For the hierarchical lattice, the overlap has a simple interpretation. We have seen that, at least for large times, most of the activity essentially takes place along the same path. However, from time to time, the minimal path jumps from one conformation to another, whose distance to the previous one is quantified by the Hamming distance. The scarcity of the jumps allows us to neglect the occurrence of simultaneous multiple jumps. Let us define the probability, $P_{L}(d, t)$, as the probability that a jump equal to $d=2^{n}$ takes place, i.e. the probability that the current path differs from the previous one by $d$ sites in a system of size $L=2^{N}$. This quantity, apart from containing information about how the average value of $d$ changes with time, is also the natural analogue of an "avalanche distribution" in this model. As will be seen further down, this quantity does indeed decay for large times as a power-law of the distance $d$ like in many other self-organized critical models. However the distribution has a time-dependent prefactor unlike other models with a true steady state.

For $n=N$, we have to consider a jump at the largest scale available in the system. At this level, the lattice can be coarse-grained as a generation 1 lattice. The probability for such a jump to occur is equal to the probability that in a two-site model, the activity moves from one site to the other one. We show in the appendix that this probability, is equal to $1 /(t+1)$, and thus for large times,

$$
P_{L}\left(d=2^{N}, t\right) \approx 1 / t
$$

Let us now consider a smaller jump size, i.e. $n=$ $N-1$. This means that one half of the actual minimal path should move to a different configuration. Thus we focus on a subsystem of size $L / 2$, whose age is $T$. In the appendix, we show that the probability for such an age is $q(T, t)=2(T+1) /(t+1)(t+2) \approx 2 T / t^{2}$, (see Eq. A4). Moreover, we have two such subsystems in series and thus the probability that the Hamming distance is $L / 2$ in a system of size $L$ is approximately twice the probability 


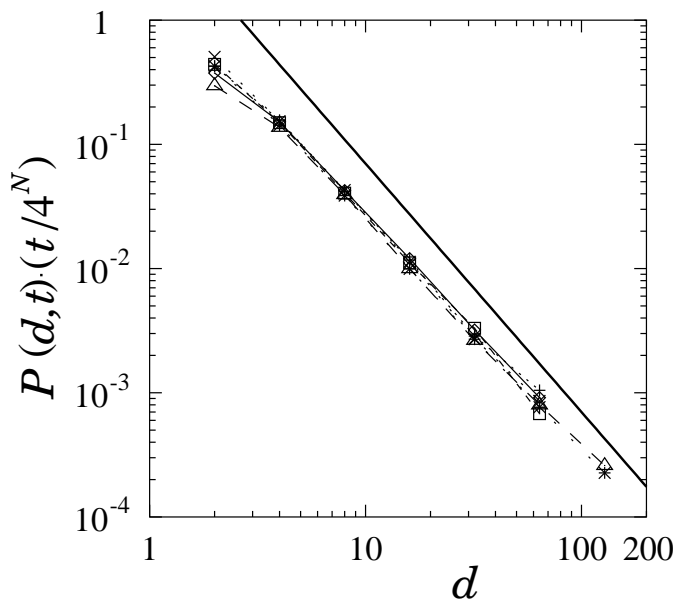

FIG. 6: $P(d, t) t$ scaled by the system size for the hierarchical lattice of generation $N=6$ at times $t / L=100,200,300$ and 1000 and for generation $N=7$ for times $t / L=100$ and 500 . Time is measured in terms of the system size and the straight line has a slope -2 .

that in one subsystem the Hamming distance is equal to the system size, $P_{L / 2}(L / 2, t)$. Integrating over all times $T$ with the above probability we have

$$
P_{L}(L / 2, t) \approx 2 \int_{0}^{t} \frac{2 T}{t^{2}} \frac{1}{T} \mathrm{~d} T=4 / t
$$

For smaller jumps, we can repeat the same argument recursively, to obtain

$$
\begin{aligned}
P_{L}\left(2^{n}, t\right) \approx & 2^{N-n} \int_{0}^{t} \int_{0}^{T_{1}} \cdots \int_{0}^{T_{N-n+1}} \frac{2 T_{1}}{t^{2}} \frac{2 T_{2}}{T_{1}^{2}} \cdots \times \\
& \times \cdots \frac{2 T_{N-n}}{T_{N-n+1}^{2}} \mathrm{~d} T_{1} \cdots \mathrm{d} T_{N-n}= \\
= & \frac{2^{2(N-n)}}{t}=\frac{L^{2}}{t d^{2}}
\end{aligned}
$$

As one can see this result agrees well with the numerical results shown on Fig. 6. Moreover, this expression is to be compared with the Hamming distribution obtained with a logarithmic measure for $d$ on the Euclidean lattice [5], which has the same functional form. Hence this distribution is the same for the two lattices despite their connectivities being very different. Note also that this quantity scales perfectly with the system size and is described at all times by the reduced time distribution $t / L$ as evidenced in Fig. 3. Thus the behaviour of the Hamming distance is much simpler than the slow density increase in the system.

\section{DISCUSSION AND CONCLUSION}

We have presented simulation results and an asymptotic analysis of the behavior of the the optimization and restructuring model on the hierarchical lattice. The two lattices are very different in structure, yet they also exhibit remarkably similar features.

The Hierarchical lattice is easier to analyse due to its recursive structure. For example, the very specific connectivity of sites on this lattice ensures that large jumps are always possible, though rare at late times. In the Euclidean lattice, these are strongly suppressed by a further feedback effect: the localization of the path limits the density increasing effect of the dynamics to a small region around the path which in turn intensifies the localization. This feature also results in the density map being very different in the two cases. In the Euclidean case [5] the inhomogeneities in a late-time snap shot of the system are much more enhanced. Another difference is that changes in conformation in the hierarchical lattice are organized in a strictly hierarchical way. This is clearly not so on the square lattice, where randomness and self-organization play an important role. Nevertheless, the overall behavior of the two type of lattices is remarkably similar.

These similarities are most apparent in the time and size dependence of the average density (Fig. 2). In both cases we have a data collapse for short times, while for longer times the dynamics becomes slower and slower as the size of the system increases. For the hierarchical lattice, we have obtained an analytic expression for the scaling of the Hamming distance, for the local age distribution, and its multifractal spectrum, and the asymptotic average density evolution with time. In particular, the mechanism behind the breakdown of ergodicity, and the unusual size dependence of the density evolution can be traced back to the multifractal distribution of age. The latter provides a novel scenario for "glassy" ageing.

\section{ACKNOWLEDGMENTS}

This work was supported by EPSRC, UK, OTKA T029985, T035028. SK would like to thank Robin Stinchcombe for useful discussions.
[1] L.E. Reichl A modern course in statistical physics, 2nd edition, Wiley, New York, (1998)

[2] J. P Bouchaud, L. F. Cugliandolo, J. Kurchan, M.
Mezard, Spin glasses and random fields Ed A. P Young, (1998), M. R. Evans, e-print cond-mat/0111171.

[3] Per Bak, How Nature Works: The Science of Self- 
Organized Criticality, Copernicus, New York (1996).

[4] J. Török, S. Krishnamurthy, J. Kertész and S. Roux, Phys. Rev. Lett. 84, 3851 (2000).

[5] J. Török, S. Krishnamurthy, J. Kertész and S. Roux, Shearing of loose qranular materials: A mesoscopic model, e-print cond-mat/0209209.

[6] S. Luding, M. Nicolas and O. Pouliquen, page 241 in Compaction of Soils, Granulates and Powders, D. Kolymbas and W. Fellin eds., (A. A. Balkema, Rotterdam 2000), also at e-print cond-mat/0003172.

[7] A. N. Berker and S. Ostlund, J. Phys. C 12, 4961 (1979).

[8] S. Kirkpatrick in Ill Condensed Matter, Eds R. Balian, R. Maynard and G. Toulouse North-Holland Publishing (1979).

[9] A. Rosas and S. Coutinho, e-print cond-mat/0107407.

[10] J. Cook and B. Derrida, J. Stat. Phys. 57, 89 (1989); E. Medina and M. Kardar, J. Stat. Phys. 71, 967 (1993).

[11] M. Kardar, Phys. Rev. Lett. 55, 2923 (1985); T. HalpinHealy and Y.-C. Zhang, Phys. Rep. 254, 215 (1995).

[12] P. Bak and K. Sneppen, Phys. Rev. Lett. 71, 4083 (1993).

[13] K. Sneppen, Phys. Rev. Lett. 69, 3539 (1992).

[14] M. Paczuski, S. Maslov and P. Bak, Phys. Rev. E 53, 414 (1995).

[15] J. Török, S. Krishnamurthy, J. Kertész and S. Roux, Euro. Phys. J. B 18 697, (2000).

[16] E. J. Gumbel, The Statistics of Extremes Columbia University Press, NY 1958; J. Galambos, The Asymptotic Theory of Extreme Order Statistics, (Wiley, New York 1978).

[17] H. J. Herrmann and S. Roux, Statistical Models for the Fracture of Disordered Media, (Elsevier Science, New York 1990); P. L. Krapivsky, E. Ben-Naim and I. Grosse, e-print cond-mat/0108547.

[18] P.L. Krapivsky and E. Ben-Naim, J. of Chem. Phys. 100, 6778 (1996); A. J. Kolan, E. R. Nowak and A. V. Tkachenko, Phys. Rev. E 593094 (1999); S. F. Edwards and D. Grinev, e-print cond-mat/9905114

[19] J. B. Knight, C. G. Fandrich, C. N. Lau, H. M. Jaeger and S. R. Nagel, Phys. Rev. E 51, 3957 (1995).

[20] E. R. Nowak, J. B. Knight, E. Ben-Naim, H. M. Jaeger and S. R. Nagel, Phys. Rev. E 57, 1971 (1998).

[21] G.C. Barker and A. Mehta, Phys. Rev. E 47, 184 (1993).

[22] T. Boutreux and P.G. de Gennes, Physica A 244, 59 (1997).

[23] G. Peng and T. Ohta, Phys. Rev. E. 57, 829 (1998).

[24] E. Ben Naim, J. B.Knight, E.R. Nowak, H. M. Jaeger and S. R. Nagel, Physica D 123, 380 (1998).

[25] J. J. Brey, A. Prados, J. Phys: Condens. Matter 14, 1489 (2002).

[26] J. Talbot, G. Tarjus and P. Viot, Phys. Rev. E 61, 5429 (2000).

[27] D. A. Head, Phys. Rev. E 62, 2439 (2000).

[28] S. N. Majumdar, D. S. Dean and P. Grassberger, Phys. Rev. Lett. 86, 2301 (2001).

[29] E. Caglioti, V. Loreto, H. J. Herrmann and M. Nicodemi, Phys. Rev. Lett. 79, 1575 (1997).

[30] M. Nicodemi, A. Coniglio and H. J. Herrmann, Phys. Rev. E 55, 3962 (1997).

[31] A. Baldassarri, S. Krishnamurthy, V. Loreto and S. Roux, Phys. Rev. Lett. 87, 224301 (2001).

[32] R. B. Stinchcombe and M. Depken, Phys. Rev. Lett. 88 125701, 2002.

[33] C. Monthus and J.P. Bouchaud, J. Phys. A: Math. \& Gen. 29, 3847, (1996).
[34] P. Le Doussal, C. Monthus and D. S. Fisher, Phys. Rev. E 59, 4795 (1999).

[35] P. Sollich and M. R. Evans, Phys. Rev. Lett. 83, 3238 (1999).

[36] S. M. Fielding, P. Sollich and M. E. Cates, Journal of Rheology 44, 323 (2000).

[37] F. Ritort, Phys. Rev. Lett. 75, 1190 (1995).

[38] The problem is hence identical to that of finding the extremum of a set of $N$ independent identically distributed random variables where $N=t+1$. Various results for the extremum, in the context of weather records, are presented pedagogically in B. Schmittmann and R. K. P. Zia, Am. J. Phys. 67, 1269 (1999).

\section{APPENDIX A: TIME PARTITIONING IN THE TWO-SITE PROBLEM}

We consider here the two-site model and prove that after the elapse of a time $t$, the probability that a given site has been visited $T$ times is uniformly distributed between 0 and $t$. The following proof is valid for any distribution $p(x)$. Let us compute $q(T, t)$, the probability that the active site has been refreshed $T$ times up to time $t$. Let the value of the recently refreshed site be denoted by $x$ and the inactive site at $t$ be $b_{t}$. At time $t$ two things may happen:

i) either $x<b_{t-1}$, and thus $T_{t}=T_{t-1}+1$. This happens with probability

$(t+1) /(t+2)$

ii) or $x>b_{t-1}$, and thus $T_{t}=t-T_{t-1}$ with probability $1 /(t+2)$.

Note that for any distribution $p(x)$ the activity change can only be due to the fact that the largest generated random number up to time $t$ is at instant $t$. This happens with probability $1 /(t+2)$ because in an independent time series of random numbers the largest number is equally likely to be anywhere. At time $t=0$ we have to initialize the system by generating two random numbers for the two sites. This is the reason for the shift in time from $t$ to $t+2$.

Now we can write down a simple evolution equation for $q(T, t)$

$$
q(T, t)=q(T-1, t-1) \frac{t+1}{t+2}+q(t-T-1, t-1) \frac{1}{t+2}
$$

The general solution of the recursion can be written as

$$
q(T, t)=A \frac{(T+1)}{(t+1)(t+2)}+B
$$

It is simple to compute $q(t, t)$ from the above recurrence, and get

$$
q(t, t)=\frac{2}{(t+2)}
$$


and thus $B=0$ and $A=2$. Thus finally

$$
q(T, t)=\frac{2(T+1)}{(t+1)(t+2)}
$$

Now, the number of updates of the other site is simply $q(t-T, t)$, thus we can formulate the probability distribution that a site has been updated $T$ times:

$$
\begin{aligned}
r(T, t) & =\frac{1}{2}(q(T, t)+q(t-T, t))= \\
& =\frac{(T+1)+(t-T+1)}{(t+1)(t+2)}=\frac{1}{(t+1)}
\end{aligned}
$$

which is independent of $T$. Thus $r(T, t)$ is uniform.

\section{APPENDIX B: AVERAGE DENSITY OF THE INACTIVE SITE IN THE TWO SITE MODEL}

Since the hierarchical lattice can be considered as a set of two-site systems, we need to consider the distribution of the maximum in a two site model in the case when each of the sites is taken from a distribution $p_{n}(x)$. Here the subscript $n$ denotes that this is the distribution for a sum of $n$ independent random numbers each of which is taken from the uniform distribution. We need only consider the case when $n=2^{N}$ as the length of the hierarchical lattice can only be of this form.

Unfortunately, $p_{n}(x)$ is difficult to formulate in a general way. For large $n$ this is a Gaussian for moderate values of $x$. However very close to the extremes 0 and 1 , it is a power-law as we will see below. It is this regime which is asymptotically reached and hence relevant for our purposes. We hence only consider the regimes $x<1 / n$ or $x>1-1 / n$ (cases when only one number out of $n$ may reach its extreme value 1 in the $x<1 / n$ case and 0 in the $x>1-1 / n$ case).

Let us recall the formula for the average value of the largest generated number up to time $t$ when each of the individual numbers $x$ is taken from a distribution $p_{n}(x)$ :

$$
B^{(n)}(t)=(t+1) \int_{0}^{1} x p_{n}(x) P_{n}^{t}(x) \mathrm{d} x,
$$

where $P_{n}(x)$ is the cumulative distribution of $p_{n}(x)$. Thus $P_{n}^{t}(x)$ accounts for the probability that the $t$ other numbers are less than $x$. The $t+1$ factor is needed to take into account the fact that the position of the largest number can be anywhere in time. The index $n$ indicates that the distributions describe the average of $n$ independent, uniformly distributed random numbers.

For $t \gg 1$ we have $P_{n}^{t}(x) \ll 1$ for most values of $x$, except for a $1 / n$ neighborhood of 1 .

So in the integration the most important contribution comes from the part that is close to 1 . This permits us to restrict the integral to the part that we can calculate

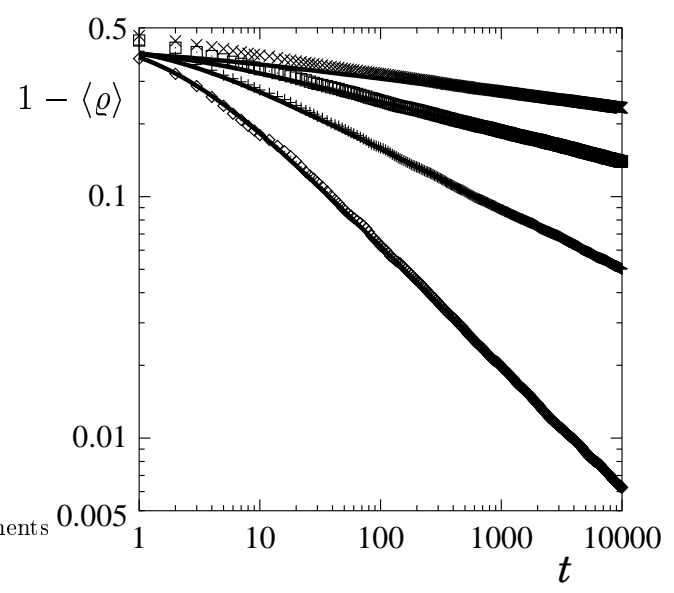

FIG. 7: The test of the results in Eq. B7 against numerical simulations on the same model. The solid lines are the analytical solutions, the symbols indicate numerical simulation results. The system sizes are $n=2(\diamond), 4(+), 8(\square), 16(\times)$

without loss of consistency:

$$
B^{(n)}(t) \simeq \int_{1-1 / n}^{1}(t+1) x p_{n}(x) P_{n}^{t}(x) \mathrm{d} x \quad(t \gg 1)
$$

The probability distribution close to the limits takes the following forms

$$
\begin{aligned}
p_{n}(x)_{x<1 / n} & =\frac{n^{n}}{(n-1) !} x^{n-1} \\
\left.p_{n}(x)\right|_{x>1-1 / n} & =\frac{n^{n}}{(n-1) !}(1-x)^{n-1}
\end{aligned}
$$

The cumulative distribution is the integral of the above:

$$
\left.P_{n}(x)\right|_{x>1-1 / n}=1-\frac{n^{n-1}}{(n-1) !}(1-x)^{n}
$$

Let us now turn back to Eq. B2. Using an $x=1-y$ variable replacement and doing integration in parts we arrive at the following formula after neglecting the exponentially decaying parts:

$$
\begin{aligned}
B^{(n)}(t)= & (t+1) \frac{n^{n}}{(n-1) !} \int_{0}^{1 / n}(1-y) y^{n-1} \times \\
& \times\left(1-\frac{n^{n-1}}{(n-1) !} y^{n}\right)^{t} \mathrm{~d} y= \\
= & 1-\int_{0}^{1 / n}\left(1-\frac{n^{n-1}}{(n-1) !} y^{n}\right)^{t+1} \mathrm{~d} y
\end{aligned}
$$

We rewrite the integrand in a $(\cdots)^{t+1} \equiv \exp \{(t+$ 1) $\log (\cdots)\}$ form and make a Taylor expansion in $y^{n}$ around $y=0$ to the second order. The result can be written in the following form that we use in our calcula- 
tions:

$$
\begin{aligned}
B^{(n)}(t)= & 1-\Gamma(1 / n)(n !)^{1 / n} n^{-2}\left(t+\frac{3 n+1}{2 n}\right)^{-1 / n}+ \\
& +\mathcal{O}\left(\frac{1}{t^{1 / n+1}}\right) \sim \frac{1}{e} t^{-1 / n}
\end{aligned}
$$

On Fig. 7 we can see that the above approximation is excellent for small $n$ and large $t$. 